\title{
Binarization of MRI with Intensity Inhomogeneity using K- Means Clustering for Segmenting Hippocampus
}

\author{
K. Somasundaram ${ }^{1}$ and T. Genish ${ }^{2}$ \\ ${ }^{1}$ Image Processing Lab, Department of Computer Science and Applications, Gandhigram \\ Rural Institute, Gandhigram, Tamil Nadu, India. \\ ka.somasundaram@gmail. com \\ ${ }^{2}$ Image Processing Lab, Department of Computer Science and Applications, Gandhigram \\ Rural Institute, Gandhigram, Tamil Nadu, India. \\ genishit@gmail.com
}

\begin{abstract}
Medical image segmentation plays a crucial role in identifying the shape and structure of human anatomy. The most widely used image segmentation algorithms are edge-based and typically rely on the intensity inhomogeneity of the image at the edges, which often fail to provide accurate segmentation results. This paper proposes a boundary detection technique for segmenting the hippocampus (the subcortical structure in medial temporal lobe) from MRI with intensity inhomogeneity without ruining its boundary and structure. The image is pre-processed using a noise filter and morphology based operations. An optimal intensity threshold is then computed using K-means clustering technique. Our method has been validated on human brain axial MRI and found to give satisfactory performance in the presence of intensity inhomogeneity. The proposed method works well even for weak edge. Our method can be used to detect boundary for accurate segmentation of hippocampus.
\end{abstract}

\section{KEYWORDS}

Segmentation, intensity inhomogeneity, hippocampus, morphological operations, $k$-means, thresholding, $M R I$

\section{INTRODUCTION}

Intensity inhomogeneity [1] often occurs in real-world images due to various factors, such as spatial variations in illumination and imperfections in imaging devices, which complicates many problems in image processing and computer vision. Image segmentation may become considerably difficult for images with intensity inhomogeneity overlapping with the ranges of the intensities in the regions to be segmented. This makes it impossible to identify these regions based on the pixel intensity. The widely used image segmentation algorithms [2] [3] usually rely on intensity homogeneity, and therefore are not applicable to images with intensity inhomogeneity. In general, intensity inhomogeneity has been a challenging task in image segmentation.

One of the important stages in medical image analysis is segmentation of objects or identification of their contours. Medical images are relatively hard to segment due to several undesirable properties such as low signal-to-noise and contrast-to-noise ratios, and discontinuous edges. The segmentation of the hippocampus (critical structure in human limbic system, responsible for memory forming, organizing, and storing) is a difficult task [4] [5] due to its small size, low 
The International Journal of Multimedia \& Its Applications (IJMA) Vol.5, No.1, February 2013

contrast, and apparent discontinuity of the edges. Some previous works have utilised manual thresholding [6] [7], annealing-based optimal threshold determining method [8] [9], image intensity standardization for correcting acquisition-to-acquisition signal intensity variations [10] [11] [12], homomorphic methods [13], curvelet and wavelet based methods [14] [15] [16] [17]. However such methods sometimes are sensitive to small data variations and it is difficult to find the optimum threshold value for segmenting the hippocampus from brain regions. In order to overcome this problem, the image is first pre-processed by mean filter followed by grey-scale morphological operations to remove or subdue the background noise. Since mean filter is consistently more effective than median-based algorithms [11] for removing intensity inhomogeneity in MR images, it is used in our proposed scheme. The filtered image is then binarized using the threshold value computed by K-means clustering technique [18] [19]. Application of our method on few MRI of human head scans show that our approach gives improved results. The remaining paper is organized as follows. In Section 2, the proposed scheme is explained. In section 3, the details of materials used are given. In section 4, we present the results and discussions. In Section 5, the conclusions are given.

\section{PROPOSED METHOD}

The first step in the proposed method is to remove the noise. We apply mean filter to the input image to remove noise. We consider a small mask of size $3 \times 3$ pixels and the computation is made from top-left to bottom-right of an image to remove the background noise in the MRI. Then, the grey-scale morphology based top-hat and bottom-hat filtering are used to smooth the pixel intensities of the image to correct uneven background. Generally morphological operations are used for binary images to dilate or to shrink. But, here we perform morphological operations on grey-scale images to make the darker regions as lighter and vice-versa. After performing morphological top-hat and bottom-hat filtering, the region of interest (hippocampus) appeared as a darker part. In this operation, the boundary of the region is not affected. The flow chart of the proposed scheme is shown in Figure 1.

The top-hat filtering is given as:

$$
I_{\text {top }}=f-\left(A^{\circ} B\right)
$$

and the bottom-hat filtering is given as:

$$
\mathrm{I}_{\mathrm{bot}}=\mathrm{f}-(\mathrm{A} \cdot \mathrm{B})
$$

where, $\mathrm{f}$ is the original image, $\mathrm{A}{ }^{\circ} \mathrm{B}$ and $\mathrm{A} \bullet \mathrm{B}$ are the morphological opening and morphological closing of an image A by a structuring element B. Since the appearance of the hippocampus is small, structuring element $\mathrm{B}$ of size $3 \times 3$ is used. The filtered image is then obtained by:

$$
I_{F}=\left(f+I_{t o p}\right)-I_{b o t}
$$

\subsection{Generating binary image}

We have tested several methods to generate binary images. The examined methods include (a) Otsu`s thresholding technique (b) Ridler`s Calvard method and (c) K-Means clustering (KM). The Otsu`s thresholding technique involves iterating through all the possible threshold values and calculating a measure of spread for the pixel levels each side of the threshold. Ridler`s Calvard chooses an initial threshold and then iteratively calculates the next one by taking the mean of the average intensities of the background and foreground pixels determined by the first thresholding, repeating this until the threshold converges. $\mathrm{KM}$ is an unsupervised clustering method that the user should specify the number of clusters. 


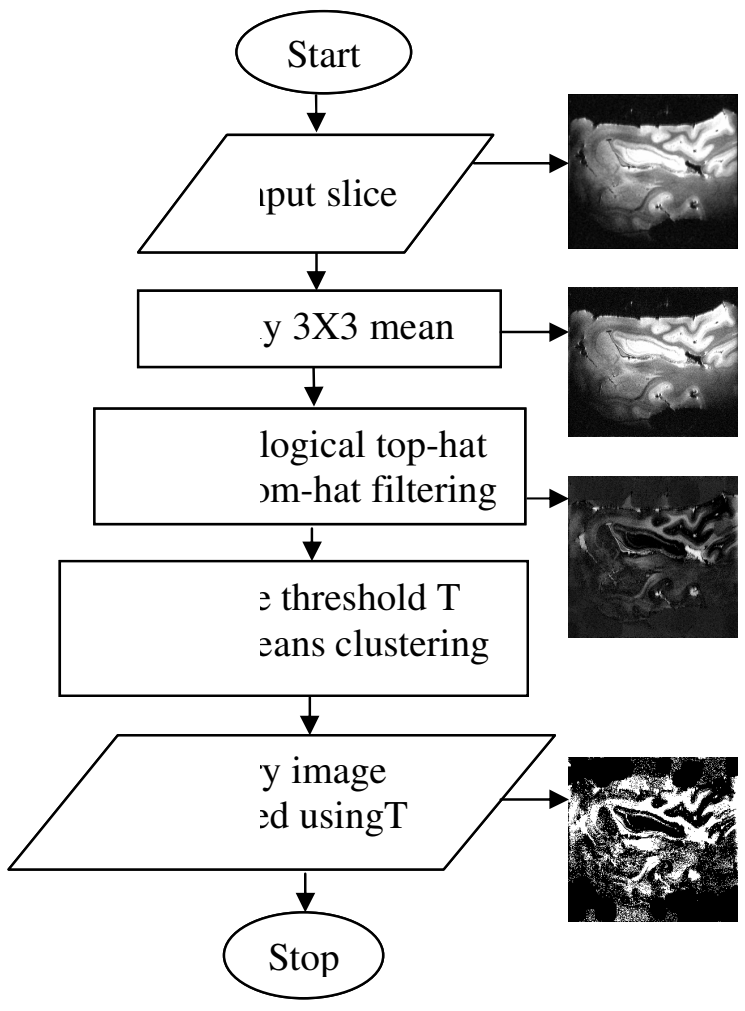

Figure 1. Flow chart of the proposed method.

The criteria we use to assess the binary images is focused on the hippocampus region which aims the separation of grey matter from the white matter. Among the thresholding techniques described above, the KM meets the demand and hence it is used to compute threshold value in the proposed method. The Figure 2. shows the results of binary images generated by Otsu`s, Ridler Calvard and $\mathrm{KM}$.

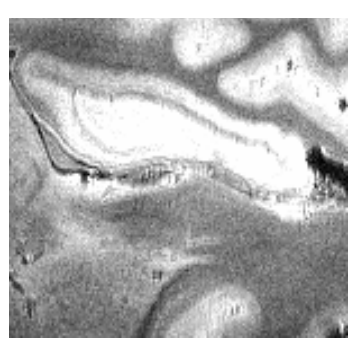

(a)

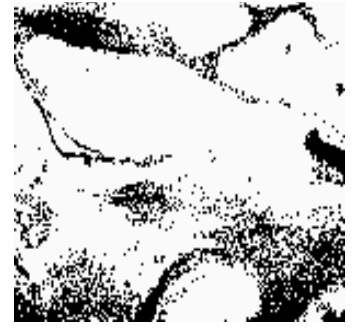

(b)

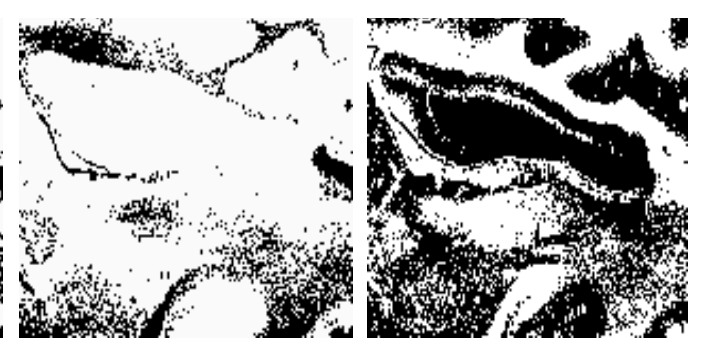

(c)

(d)

Figure 2. (a) Original image. Binary images obtained by(b) Otsu`s (c) Ridler`s Calvard and (d) KM.

From the above figure, we can observe that the binary image generated by KM produces satisfactory result when compared to other two methods.

\subsection{Finding Threshold using KM Clustering}

K-Means algorithm is an unsupervised clustering algorithm that classifies the input data points into multiple classes based on their inherent distance from each other. The algorithm assumes that 
The International Journal of Multimedia \& Its Applications (IJMA) Vol.5, No.1, February 2013

the data features form a vector space and tries to find natural clustering in them. The algorithm consists of the following steps to classify a data set $\mathrm{x}_{\mathrm{i}}, \mathrm{i}=1,2,3, \ldots \mathrm{n}$ into $\mathrm{k}$ clusters.

Step 1: Initialize the centroids $C_{j}$ with $\mathrm{k}$ random intensities.

Step 2: Assign each data point $\mathrm{x}_{\mathrm{i}}$ to the group that has the closest centroid.

Step 3: When all the data points are assigned to any of the cluster, calculate the positions of $\mathrm{k}$ centroids.

Step 4: Repeat steps 2 and 3 until the cluster labels of the image does not change anymore.

This algorithm aims at minimizing an objective function given by:

$$
\mathrm{F}=\sum_{j=1}^{k} \sum_{i=1}^{n}\left\|x_{i}^{(j)}-C_{j}\right\|^{2}
$$

where, $\left\|x_{i}^{(j)}-C_{j}\right\|^{2}$ is a measure of intensity distance between a data point $\mathrm{x}_{\mathrm{i}}$ and the cluster center $\mathrm{C}_{\mathrm{j}}$. In the proposed method, the value of $\mathrm{k}$ is assumed as 3 . The total mean value of the image $\mathrm{I}_{\mathrm{F}}$ and its doubled, tripled products are assigned as an initial centroids. The final clusters obtained by the k- means algorithm $M_{1}, M_{2}$ and $M_{3}$ are used to compute the threshold value $\mathrm{T}$ given by:

$$
\mathrm{T}=\left(M_{1}+M_{2}+M_{3}\right) / 2
$$

The given input image $I_{F}(x, y)$ is converted to a binary image $g$ as:

$$
\begin{aligned}
\mathrm{g}(\mathrm{x}, \mathrm{y}) & =1 \text { if } \mathrm{I}_{\mathrm{F}}(\mathrm{x}, \mathrm{y})>=\mathrm{T} \\
& =0 \text { otherwise }
\end{aligned}
$$

\section{MATERIALS USED}

The materials used for our experiment are obtained from Neuroimaging Informatics Tools and Resources Clearinghouse (NITRC) [20] that facilitates neuroimaging resources for functional and structural neuroimaging analyses. In NITRC, Paul Yushkevich posted list of high-resolution postmortem MRI of the human hippocampus entitled Penn Hippocampus Atlas (PHA). The T2weighted (T2w) MRI data was acquired from 9.4T scanner. It consists of 130 axial $0.2 \mathrm{~mm}$ slices of dimension 300 X 280 pixels. Among them, the slices numbered from Penn002L_01078 to Penn002L_01092 are considered for experiments because the hippocampus is not present in other slices.

\section{RESULT AND DISCUSSIONS}

To evaluate the performance of our method, we carried out experiments by applying our method on the material pool. Figure 3. shows a sampled slice binarized image using the threshold value $\mathrm{T}$ and bottom-hat and top-hat filtered image using T. Fig. 3. shows the result of our proposed method for all the slices. By comparing the Column 1 and Column 3, it can be observed that the contrast of an image is enhanced such that the brighter part becomes darker and vice versa. The morphological filtering produces notable results to separate the hippocampus (highlighted in Figure. $3 \mathrm{~b}$ ) from its neighbouring structures. However, when the $\mathrm{T}$ value is applied to the nonfiltered images, all the structures get collapsed and the hippocampus is seen nowhere. Since the edge plays a crucial role in image segmentation, the proposed scheme can be used as a preprocessing technique to find the edges of hippocampus. 
The International Journal of Multimedia \& Its Applications (IJMA) Vol.5, No.1, February 2013

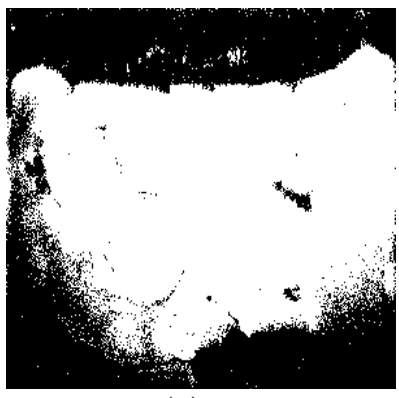

(a)

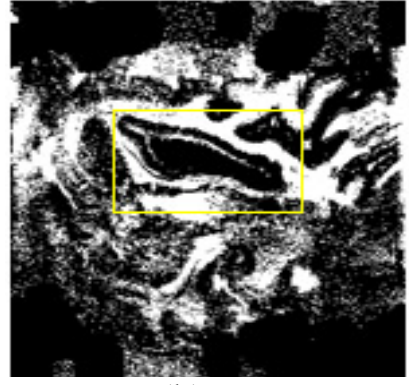

(b)

Figure3. The binarized image (a) binarization of original image using $\mathrm{T}$ (b) binarization of tophat and bottom-hat filtered image using $\mathrm{T}$.

The $3 \times 3$ mask is considered in the morphological filtering since the shape of the hippocampus resembles an oval structure. The binarized image produces satisfactory result and shows that hippocampus region does not merge or join with the adjacent regions in most of the cases.
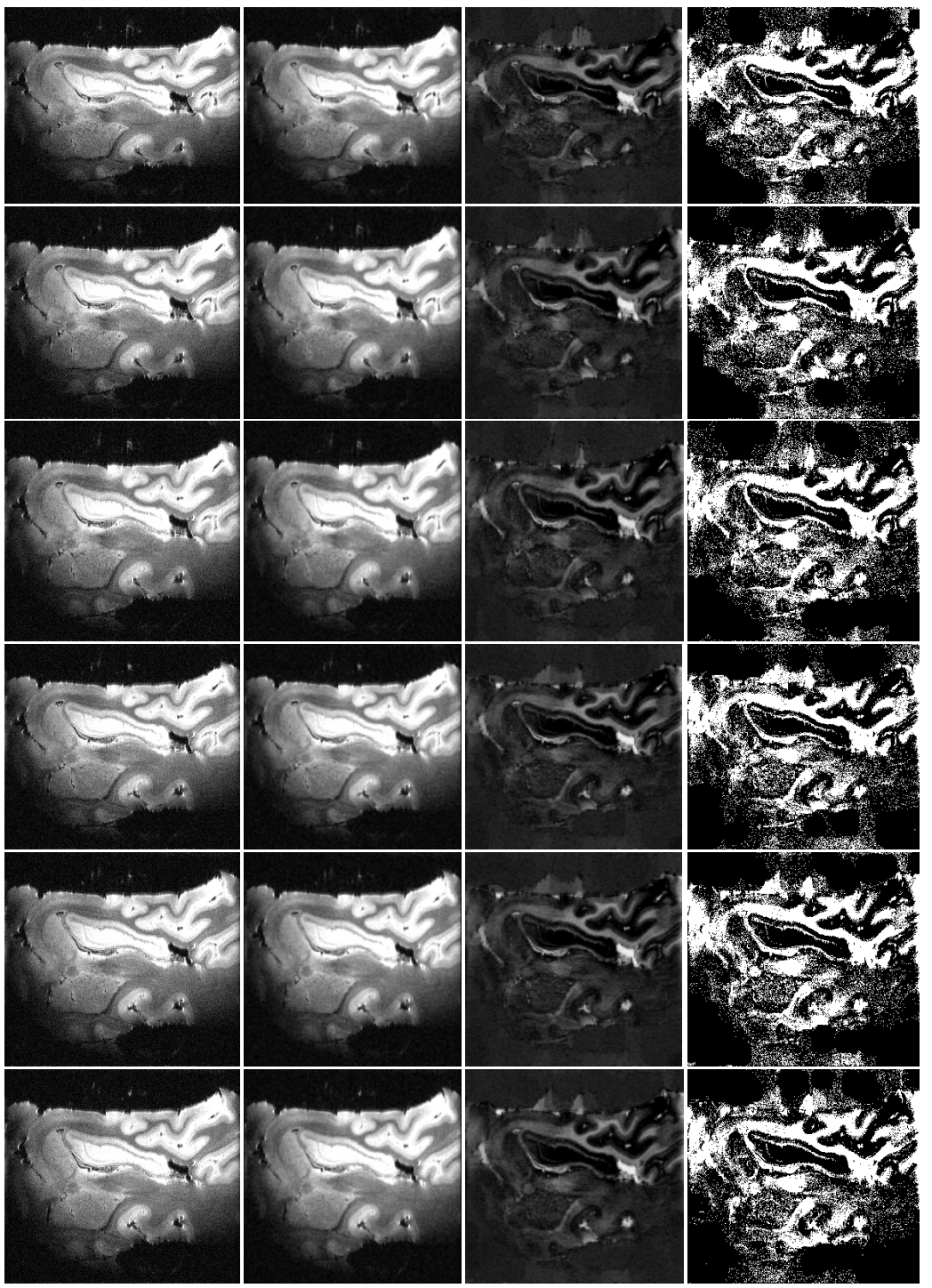
The International Journal of Multimedia \& Its Applications (IJMA) Vol.5, No.1, February 2013
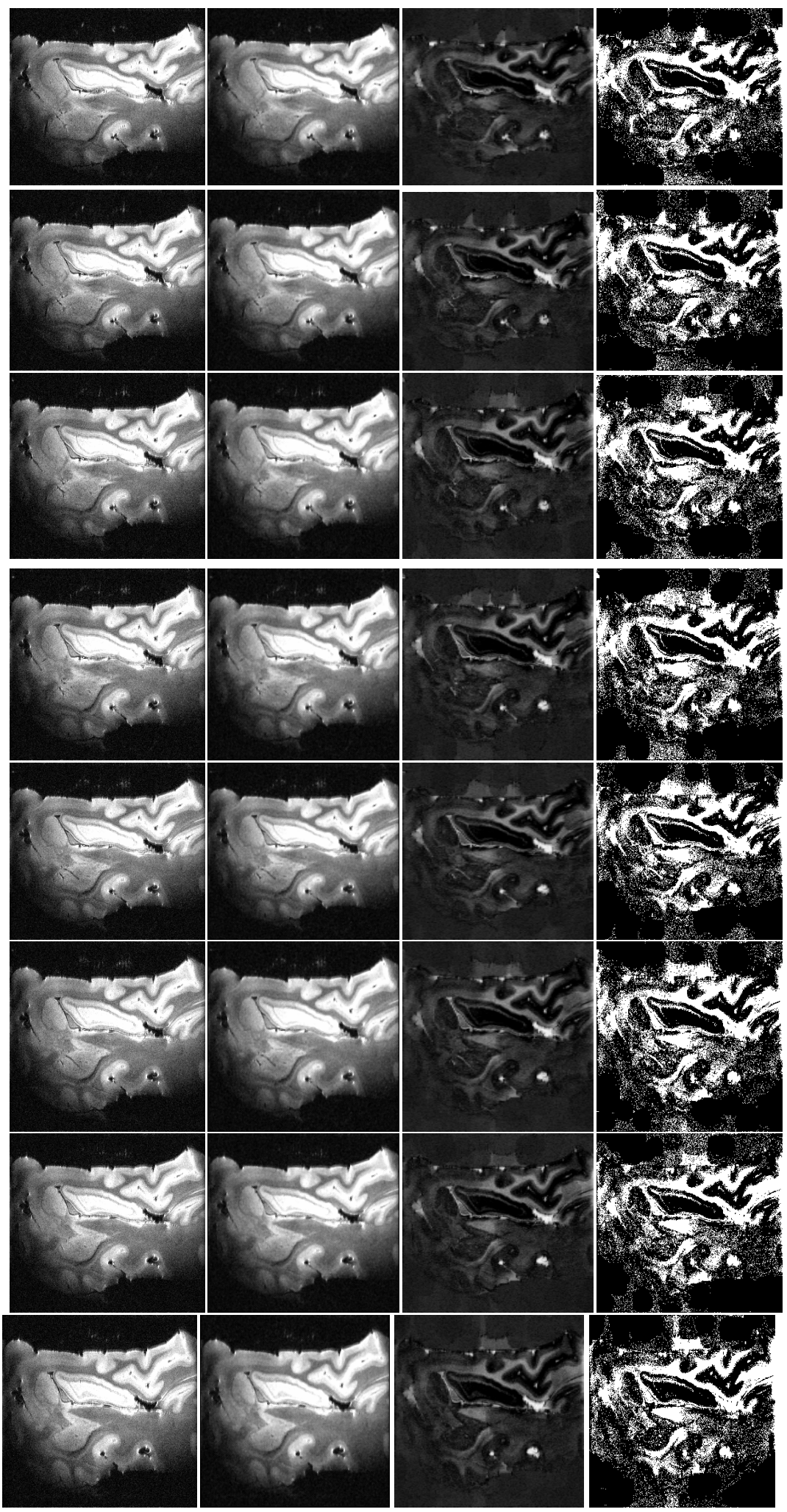
The International Journal of Multimedia \& Its Applications (IJMA) Vol.5, No.1, February 2013
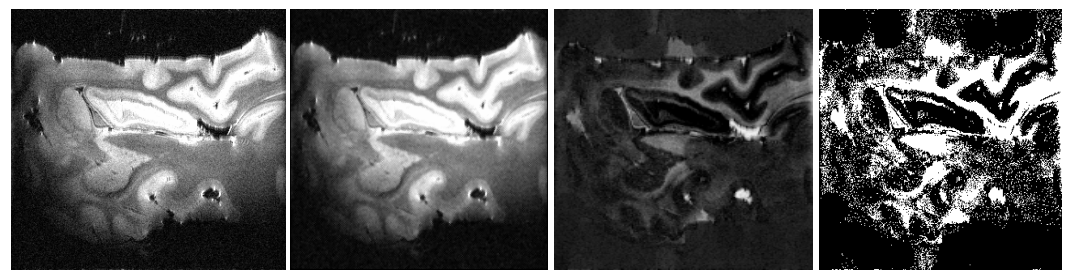

Figure 4. Image binarization using the proposed scheme (a) Original image (b) Image obtained after mean filtering (c) Image obtained after morphological filtering (d) Binarized image using kmeans clustering.

\section{CONCLUSION}

In this paper, we have proposed a binarization technique for correcting intensity inhomogeneity in MR images to remove the artifacts. The combination of morphological top-hat and bottom-hat filtering operations and k-means clustering technique are used to find edges sharply. The edges identified by our method can be used as a pre-processing technique to avoid artifacts and can be used to develop a generalized method to segment the hippocampus automatically from the human brain MRI.

\section{ACKNOWLEDGMENTS}

This work is supported by a research grant by University Grants Commission, New Delhi, India. Grant No: M.R.P, F.No-37- 154/2009(SR).

\section{REFERENCES}

[1] Chumming Li, Huang, R. Ding, Z. Chris Gatenby, J. \& Metaxas, D.N, (2011) “A Level Set Method for Image Segmentation in the Presence of Intensity In homogeneities With Application to MRI", IEEE Transactions on Image Processing, Vol. 20, No. 7, pp2007-2016.

[2] Chan, T. \& Vese, L, (2001) “Active contours without edges", IEEE Transactions on Image Processing,Vol. 10, No. 2, pp266-277.

[3] Ronfard R, (1994) "Region-based strategies for active contour models",International Journal of Computer Visio,. Vol. 13, No. 2, pp229-251.

[4] Shen, D. Susan, M.S. Resnick, M. \& Davatzikos, C, (2002) "Measuring Size and Shape of the Hippocampus in MR Images Using a Deformable Shape model”, NeuroImage, Vol. 15, No. 2, pp 422-434.

[5] Ghanei, A. Zadeh, H.S. \& Windham, J.P, (1998) "Segmentation of the hippocampus from brain MRI using deformable contours", Computerized Medical Imaging and Graphics, Vol. 22, No. 3, pp 203216.

[6] Freeborough, P.A. Fox, N.C. \& Kitney, R.I, (1997) "Interactive algorithms for the segmentation and quantitation of 3-D MRI brain scans", Computer Methods and Programs in Biomedicine, Vol. 53, No. 1, pp15-25.

[7] Boyes, B.J. Lewis, R.G. Schott, E.B. Frost, J.M.C. Scahill, R.I\& Fox, N.C, (2007) “Automatic calculation of hippocampal atrophy rates using a hippocampal template and the boundary shift integral", Neurobiology of Aging28 (2007), Vol. 28, No. 11, pp1657-1663.

[8] Jorge Haddock \& John Mittenthal, (1992) "Simulation optimization using simulated annealing", Computers \& Industrial Engineering, Vol. 22, No. 4, pp387-385. 
The International Journal of Multimedia \& Its Applications (IJMA) Vol.5, No.1, February 2013

[9] Ety Navon, Ofer Miller \& Amir Averbuch, (2005) "Color image segmentation based on adaptive local thresholds", Image and Vision Computing, Vol. 23, No. 1, pp 69-85.

[10] Somasundaram, K. \& Kalaiselvi, T, (2010) "Fully automatic brain extraction algorithm for axial T2weighted magnetic resonance images", Computers in Biology and Medicine. Vol. 40, No. 10,pp811822.

[11] Heckemann, R.A. Hajnal, J.V. Aljabar, P. Rueckert, D. \& Hammer, A, (2006) “Automatic anatomical brain MRI segmentation combining label propagation and decision fusion", NeuroImage, Vol. 33, No. 1, pp115-126.

[12] Thillou, C. \&Gosselin, B. Robust, (2004)“Thresholding Based on Wavelets and ThinningAlgorithms for Degraded Camera Images", Proceedings of ACIVS, tcts.fpms.ac.be.

13] Sonka, M. Hlavac \& Boyle, (2007), "Digital Image Processing and Computer Vision”, Thomson Learning Inc., Second Edition.

[14] Starck, J.L. Candes, E.J. \& Donoho, D.L, (2002) “The curvelet transform for image denoising”, IEEE Transactions on Image Processing, Vol. 11, No. 6, pp 670-684.

[15] Portilla, J. Strela, V. Wainwright, M.J.\&Simoncelli, E.P, (2003), “ Image denoising using scale mixtures of Gaussians in the wavelet domain" , IEEE Transactions on Image Processing,Vol. 12, No. 11, pp1338-1351.

[16] Saeedi, J. Moradi, M.H. \& Faez, K, (2010) “ A new wavelet-based fuzzy single and multi-channel image denoising", Image and Vision Computing, Vol. 28, No. 12, pp1611-1623.

[17] Huang, K. Wu, Z.Y. Fung, S.K. and Chan, H.Y, (2005) "Color image denoising with wavelet thresholding based on human visual system model”, Signal Processing: Image Communication, Vol. 20, No. 2, pp115-127.

[18] Li-Hong Juang \& Ming-Ni Wu, (2010) "MRI brain lesion image detection based on color-converted K-means clustering segmentation”, Measurement, Vol. 43, No. 7, pp941-949.

[19] D.A. Clausi, (2002)"K-means Iterative Fisher (KIF) unsupervised clustering algorithm applied to image texture segmentation”,Pattern Recognition, Vol. 35, No. 9, pp1959-1972.

[20] http://www.nitrc.org/projects/pennhippoatlas. 
The International Journal of Multimedia \& Its Applications (IJMA) Vol.5, No.1, February 2013

\section{Authors}

K. Somasundaram was born in the year 1953. He received the M.Sc degree in Physics from University of Madras, Chennai, India in 1976, the Post Graduate Diploma in Computer Methods from Madurai Kamaraj University, Madurai, India in 1989 and the Ph.D degree in theoretical Physics from Indian Institute of Science, Bangalore, India in 1984. He is presently the Professor and Head of the Department of Computer Science and Applications, and Head, Computer Centre at Gandhigram Rural Institute, Gandhigram, India. From 1976 to 1989, he was a Professor with the Department of Physics at the same Institute. He was previously a Researcher at an International Centre for Theoretical Physics, Trieste, Italy and a Development Fellow of Commonwealth Universities at the school of Multimedia, Edith Cowan University, Australia. His research interests are in image processing, image compression and medical imaging. He is a Life member of Indian Society for Technical Education and Telemedicine Society of India. He is also an annual member in ACM, USA and IEEE Computer Society, USA.

T. Genish was born in the year 1988. He received the B.Sc. degree in Information Technology from Madurai Kamaraj University, Madurai, India in 2005, the M.Sc(IT) degree from the Gandhigram Rural Institute- Deemed University, India in 2010. Currently he is a doing Ph.D in Medical Image Segmentation in the same university.
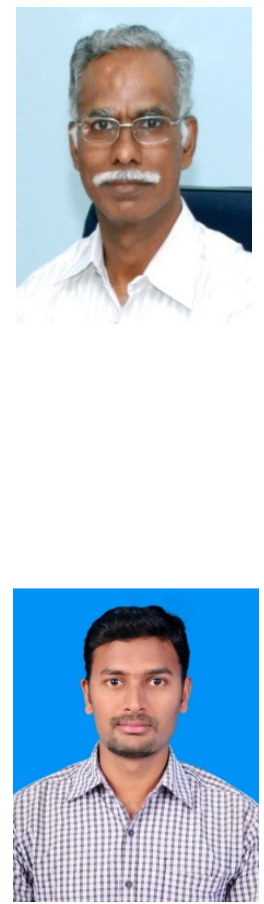